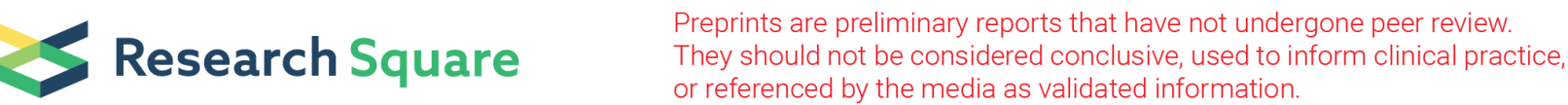

\section{One-stage anterior focus debridement, interbody graft using titamium mesh cages, and anterior instrumentation and fusion in the surgical treatment of short segment thoracic tuberculosis}

huijun zhang ( $\nabla$ zhj0525196@163.com )

xian chest hopital https://orcid.org/0000-0003-3452-0496

zenghui lu

xi'an Chest Hospital

lin wei

xi'an chest hospital

chao ding

Shanghai Jiao Tong University Affiliated Chest Hospital

junsong yang

United Hospital

Research article

Keywords: Spinal tuberculosis, anterior approach, debridement, internal fixation

Posted Date: May 29th, 2020

DOI: https://doi.org/10.21203/rs.3.rs-22042/v2

License: (c) (1) This work is licensed under a Creative Commons Attribution 4.0 International License.

Read Full License 


\section{Abstract}

Study design:This is a retrospective study

Objective:The purpose of this study was to evaluate the clinical efficacy of primary anterior debridement, bone graft fusion and internal fixation in the treatment of short segmental thoracic tuberculosis.

\section{Methods}

We performed a retrospective analysis of 16 adult patients with short segment thoracic spinal TB who underwent surgery between September 2013 to March 2017. All the 16 patients were treated using a single primary anterior debridement, bone graft fusion and internal fixation. Clinical manifestations, laboratory, neurological symptoms,bone fusion and imaging results were subjected to statistical analysis.

\section{Results}

All patients underwent operation successfully. The symptoms of chest and back pain were alleviated and disappeared at 1-6 months postoperatively.Patients were followed up for 24-48months (average, 35.6 \pm 9.6 months). Among 16 patients, no recurrence, and all the patients got bony spinal fusion within 4 to 8 months after surgery judging by spinal $\mathrm{x}$-ray film.

The preoperative ESR and CRP level of all patients were $72.6 \pm 27.5 \mathrm{~mm} / \mathrm{h}$ and $75.7 \pm 25.9 \mathrm{mg} / \mathrm{L}$, which decreased to $15.9 \pm 4.6 \mathrm{~mm} / \mathrm{h}$ and $4.7 \pm 2.0 \mathrm{mg} / \mathrm{L}$ at the final follow-up, respectively. The preoperative thoracic kyphosis angle were $18.2 \pm 4.1^{\circ}$ respectively. The corresponding postoperative angles were ameliorated significantly to $9.1 \pm 1.9^{\circ}$.

During the follow-up, spinal paraplegia was significantly improved in all patients. Neurologic status of the 16 patients with preoperative neurologic defcit was 3 with grade A recovered to normal; 4 with grade $B$ recovered to normal; 4 with grade $C$ recovered to normal; 3 with grade $A$ recovered to grade $D ; 1$ with grade $B$ recovered to grade $D ; 1$ with grade $C$ recovered to $D$.

\section{Conclusion}

The clinical results of primary anterior debridement, bone graft fusion and internal fixation in the treatment of short segmental thoracic tuberculosis with paraplegia or incomplete paralysis can be an effective and feasible treatment method.

\section{Background}

Tuberculosis (TB) is caused by Mycobacterium tuberculosis. over the past decades, the incidence of spinal tuberculosis has continued to increase due to population growth, acceleration of mobility, and HIV infection and spread [1]. Spinal tuberculosis is one of the most common extrapulmonary tuberculosis, accounting for the top tuberculosis of total bones and joints[2]. Spinal tuberculosis with paraplegia or 
incomplete paralysis accounts for about 10\% 46\% of spinal tuberculosis cases[3]. Spinal tuberculosis can result in serious consequences without proper therapy in time. Spinal tuberculosis forms abscesses, sequestrum, and tuberculous granulation tissue, which enter the spinal canal to compress the spinal cord, causing nerve damage or even paraplegia. Which were described by Sir Percival Pott in 1779. Thus, it is popularly known as Pott's spine [4]. Tuberculous spondylitis (Pott's disease), a common extrapulmonary manifestation of tuberculosis (TB), typically presents with back pain, tenderness, paraparesis/paraplegia, and various constitutional symptoms. Although treatment with powerful anti-tuberculosis drugs has been used, surgical management is also critical. Based on combination chemotherapy, active surgical treatment has been accepted and can effectively shorten the treatment cycle, promote tuberculosis cure, reduce morbidity, and improve the quality of life[5].

The anterior segment, the weight-bearing area of the vertebral column, is preferred for spinal tuberculosis infection. Destruction of the anterior column alters the biomechanics and stability of the spine, which increases the risk of kyphosis and paraplegia progression in patients [6, 7]. Determining the optimal operative method is crucial, especially for Pott's paralysis of tuberculosis of short segment thoracic vertebra. Anterior lesion removal bone-grafting fusion has always been a classic surgical procedure for the treatment of spinal tuberculosis[8],Because it not only can directly reach the lesion site with a larger operative horizon to completely remove lesions, but also anterior surgery can completely expose the lesions and such lesions can then be completely removed under direct vision to reduce the compression of the spinal cord. Bone grafting can be performed to correct kyphotic deformities and re-establish spinal stability[9], Moreover, full neural decompression, ample spinal stability reconstruction, and enough deformity correction can be achieved in one stage [10]. The anterior procedure leads to early resolution of the disease and faster fusion.Although the posterior approach, which is used routinely in spinal operation, may show some advantages, it damages the residual normal structure against spinal stability and disease healing [11]. Thus,Anterior surgery is more suitable for spinal tuberculosis paraplegia or incomplete paralysis, especially Pott's paralysis of tuberculosis of short segment thoracic vertebrae. Therefore, we investigated clinical outcomes of the anterior procedure for treating patients with tuberculosis paraplegia or incomplete paralysis of short segment thoracic vertebrae.

\section{Methods}

\section{Materials}

From September 2013 to March 2017, 16 patients (age range 23-74 years, with an average age of $46.3 \pm$ 14.5years) with paraplegia or incomplete paralysis of short segment thoracic vertebrae tuberculosis, including 7 men and 9 women, who underwent anterior debridement, bone grafting fusion and anterior fixation in our hospital enrolled the study.

Patients who met of the following conditions were selected: (i) Thoracic tuberculous with destruction of one or two segments of the vertebral body, (ii) A mild kyphotic deformity (Cobb angle $<30^{\circ}$ ), (iii) persisting back pain attributed to spinal instability and (iv) poor response to medical management,(v) the vertebral 
segment can be treated with right anterior internal fixation, (vi) the patient has developed paraplegia or incomplete paralysis,(vii)there were no contraindications for cardiac function and pulmonary function.

Exclusion criteria were: (i) multi-segmental involvement with severe destruction of vertebral bodies, (ii) the patient did not have paraplegia or incomplete paralysis, (iii) severe kyphosis (Cobb angle $>30^{\circ}$ ), (iv) previous thoracic surgery and (v) severe or active tuberculosis,(vi)there are contraindications to thoracotomy.

Spinal tuberculosis was diagnosed based on patients' symptoms (local pain and percussion pain accompanied with fever, night sweats, and neurological dysfunction), laboratory results (T-spot, tuberculosis antibody, erythrocyte sedimentation rate [ESR], and C-reactive protein [CRP]) and radiologic findings (radiography, computed tomography, and magnetic resonance imaging) and was confirmed by postoperative pathology examinations. Imaging studies showed vertebral body destruction, intervertebral space collapse, kyphosis, paravertebral abscess, and intraspinal invasion. Regarding thoracic vertebral damage, 1 patients had thoracic vertebrae 5 and 6 damage (T5-T6), 2 patients had thoracic vertebrae 6 and 7 damage (T6-T7), 2 patients had thoracic vertebrae 7 and 8 damage (T7-T8), 4 patients had thoracic vertebrae 8 and 9 damage (T8-T9), 5 patients had thoracic vertebrae 9 and 10 damage (T9-T10),2 patients had thoracic vertebrae 10 and 11 damage (T10-T11) (Table 1). The same surgeons reviewed the surgical indications and performed the procedures. The patients were evaluated preoperatively and postoperatively in terms of Frankel Grade, kyphotic Cobb angle, and bony fusion.

Table 1 Summary of the patients' data

\begin{tabular}{|c|c|c|c|c|c|c|c|c|c|c|}
\hline \multirow[t]{2}{*}{ Patient no. } & \multirow[t]{2}{*}{$\operatorname{Age}(y) / \operatorname{sex}$} & \multirow[t]{2}{*}{ Level } & \multirow{2}{*}{$\begin{array}{l}\text { Follow-up } \\
\text { (months) }\end{array}$} & \multicolumn{3}{|c|}{ Kyphosis angle $\left({ }^{\circ}\right)$} & \multicolumn{3}{|c|}{ Frankel grade } & \multirow{2}{*}{$\begin{array}{l}\text { Fusion } \\
\text { (months) }\end{array}$} \\
\hline & & & & PRE & POST & FFU & PRE & POST & FFU & \\
\hline 1 & $23 / M$ & T8-T9 & 24 & 23 & 11 & 11 & $A$ & $B$ & $\mathrm{D}$ & 4 \\
\hline 2 & $47 / F$ & T9-T10 & 36 & 12 & 8.4 & 10 & A & B & E & 5 \\
\hline 3 & $48 / F$ & T8-T9 & 48 & 21 & 10.6 & 11 & C & D & E & 5 \\
\hline 4 & $56 / M$ & T6-T7 & 48 & 18 & 10 & 10 & B & C & D & 6 \\
\hline 5 & $57 / M$ & T9-T10 & 42 & 17 & 9 & 9 & B & C & $E$ & 5 \\
\hline 6 & $37 / M$ & T5-T6 & 24 & 22 & 10.2 & 12 & A & B & D & 4 \\
\hline 7 & $42 / F$ & T7-T8 & 24 & 18 & 7.2 & 8 & $A$ & B & E & 5 \\
\hline 8 & $29 / M$ & T8-T9 & 30 & 20 & 7 & 6 & B & D & $E$ & 4 \\
\hline 9 & $32 / F$ & T10-T11 & 36 & 24 & 8.8 & 10 & C & D & E & 4 \\
\hline 10 & $48 / F$ & T10-T11 & 24 & 13 & 10.3 & 12 & C & D & E & 5 \\
\hline 11 & $45 / F$ & T9-T10 & 36 & 23 & 9.6 & 11 & A & C & D & 6 \\
\hline 12 & $55 / M$ & T9-T10 & 48 & 14 & 9.8 & 11 & B & C & E & 6 \\
\hline 13 & $24 / M$ & T9-T10 & 24 & 11 & 5.6 & 7 & A & C & E & 5 \\
\hline 14 & $62 / M$ & T8-T9 & 48 & 19 & 13 & 11 & B & D & E & 6 \\
\hline 15 & $74 / F$ & T7-T8 & 42 & 15 & 8 & 10 & C & D & D & 8 \\
\hline 16 & $61 / \mathrm{F}$ & T6-T7 & 36 & 21 & 6.4 & 6 & C & D & $E$ & 6 \\
\hline Mean values & $46.3 \pm 14.5$ (ages) & & $35.6 \pm 9.6$ & $18.2 \pm 4.1$ & $9.1 \pm 1.9$ & $9.7 \pm 1.9$ & & & & $5.3 \pm 1.1$ \\
\hline
\end{tabular}


Written informed consent was obtained from all patients, and the study protocol was approved by the Institutional Ethics Review Board of Xi'an chest Hospital.

\section{Methods}

\section{Preoperative preparation}

All patients received at least $2-4$ weeks of first-line anti-tuberculous treatment (rifampicin $0.45 \mathrm{~g}$, isoniazid $0.4 \mathrm{~g}$, pyrazinamide $1.5 \mathrm{~g}$,and ethambutol $0.75 \mathrm{~g}$ ) preoperatively, and supporting therapy and symptomatic treatment were conducted when the patient being hospitalized. Doses of anti-tb drugs were appropriately increased in patients with tuberculosis in other parts of the body, or in patients weighing more than $50 \mathrm{~kg}$. For patients with paraplegia or incomplete paralysis, surgery should be performed as early as possible.

\section{Surgical approach}

Informed consent for surgery is signed by the patients.Patients were instructed to lay in lateral position. Transthoracic patients received tracheal intubation with a double lumen tube, and the side lobe was collapsed intraoperatively. A standard lateral anterior approach was used, and an right anterior posterolateral surgical incision was made. Skin and subcutaneous tissues were dissected layer by layer using only an incision of the oblique costal margin. The right latissimus dorsi muscle and pectoralis major muscle were dissected layer by layer. Ribs opposite to the diseased vertebra are exposed, stripped and cut, and the cutoff parts were reserved as autologous bone graft. The right thoracic cavity was opened with an thoracotomy device, and the right lung was collapsed and the right spinal column series was exposed. The right diseased vertebral body and anterior fascia were examined with obvious swelling and abnormal color and the paravertebral abscess was aspirated with a syringe as a culture specimen. The anterior fascia of the diseased vertebral body was cut longitudinally and segmental vessels were ligated. Cheese-like substance, necrotic granulation tissue, dead bone particles and other lesions were completely removed, while normal vertebral bone tissue was retained. An abscess in the opposite direction was drawn and flushed repeatedly through the vertebral body defect. The compression of the spinal cord was completely relieved. The wound was washed repeatedly with saline, and $1-2 \mathrm{~g}$ of streptomycin powder was administered. Autogenous bone and graft fusion with a titanium cage strut combined with an anterior vertebral screw-plate internal fixation system were used to recover the normal spinal curvature of patients with kyphosis(Fig. 1,2). The drainage tube was placed postoperatively, and culture specimens were sent for pathological examination.

\section{Postoperative treatment}


Conventional electrocardiographic monitoring and anti-infection and anti-tuberculosis treatment were provided. A drainage tube was placed for 1-3 days and removed until the 24-h drainage flow was $<50 \mathrm{~mL}$. In addition, complete lung expansion was confirmed by radiography. The drainage time was extended in patients with penetrable pus cavities. Nutritional support was provided in patients with postoperative anemia, low serum albumin levels, or loss of appetite. The patients were required to get out of bed for 2 weeks after the operation.After hospital discharge, anti-tuberculosis therapy was maintained for 18-24 months.

\section{Evaluation of clinical outcomes}

All patients were examined clinically and radiologically at 3,6 and 12 months after surgery and then once a year.The ESR and CRP were measured to evaluate the activity of short segment thoracic vertebrae tuberculosis with paraplegia or incomplete paralysis. Roperatively and postoperatively in terms of Frankel Grade, kyphotic Cobb angle, and bony fusion were recorded to evaluate changes before and after surgery. Kyphosis angle:observing the lateral X-ray,the kyphotic angel was the angle formed by two lines obtained by joining the antero-superior and postero-superior corners of the above lesion,and the anteroinferior and postero-inferior corners of the vertebral below lesion[12].Postoperative radiographs were conducted to assess the level of bony fusion using the radiologic criteria of Bridwell[13].

\section{Statistical analysis}

Data were analyzed using the independent sample t test and SPSS statistical software (IBM Corp.) $\mathrm{p}$ values $<0.05$ were considered statistically significant.

\section{Results}

\section{Clinical results}

All patients underwent operation successfully. During the follow-up, spinal paraplegia was significantly improved in all patients. The symptoms of chest and back pain were alleviated and disappeared at 1-6 months postoperatively.Patients were followed up for $24-48$ months (average, $35.6 \pm 9.6$ months). Among 16 patients, no recurrence, and all the patients got bony spinal fusion within 4 to 8 months after surgery judging by spinal x-ray film and/or CT scan.

\section{Laboratory data}

The preoperative ESR and CRP level of all patients were $72.6 \pm 27.5 \mathrm{~mm} / \mathrm{h}$ and $75.7 \pm 25.9 \mathrm{mg} / \mathrm{L}$, which decreased to $15.9 \pm 4.6 \mathrm{~mm} / \mathrm{h}$ and $4.7 \pm 2.0 \mathrm{mg} / \mathrm{L}$ at the final follow-up, respectively (Table 2). 
Table 2 Laboratory data of all patients

\begin{tabular}{lllllll}
\hline $\mathrm{n}$ & \multicolumn{2}{l}{$\mathrm{ESR}(\mathrm{mm} / \mathrm{h})$} & \multicolumn{3}{l}{$\mathrm{CRP}(\mathrm{mg} / \mathrm{L})$} \\
\cline { 2 - 7 } & $\mathrm{PRE}$ & $\mathrm{POST}$ & $\mathrm{FFU}$ & $\mathrm{PRE}$ & $\mathrm{POST}$ & $\mathrm{FFU}$ \\
\hline 16 & $72.6 \pm 27.5$ & $46.6 \pm 24.1$ & $15.9 \pm 4.6$ & $75.7 \pm 25.9$ & $41.6 \pm 15.0$ & $4.7 \pm 2.0$ \\
\hline
\end{tabular}

PRE preoperative, FFU final follow-up, POST postoperative, ESR erythrocyte sedimentation rate, CRP C-reactive protein

\section{Neurologic function}

Neurological symptoms of 16 patients were manifested as complete paraplegia,lower limb weakness, chest sensation or related numbness and paresthesia. Neurological function was evaluated by the Frankel classification and is listed in Table 1. All patients achieved function status improvement at different degrees.Preoperative and postoperative neurological status by the Frankel score system ( $n=$ 16).During the follow-up, spinal paraplegia was significantly improved in all patients. Neurologic status of the 16 patients with preoperative neurologic defcit was 3 with grade $A$ recovered to normal; 4 with grade $B$ recovered to normal; 4 with grade $C$ recovered to normal; 3 with grade $A$ recovered to grade $D ; 1$ with grade $B$ recovered to grade $D ; 1$ with grade $C$ recovered to $D$.

\section{Radiological data}

The preoperative thoracic kyphosis angle were $18.2 \pm 4.1^{\circ}$ respectively. The corresponding postoperative angles were ameliorated significantly to $9.1 \pm 1.9^{\circ}$. At the final follow-up, whose loss of correction was only $0.6^{\circ}$. It still significantly improved in comparison to the preoperative measurements.( Table 1 )

\section{Complications}

There was three cases of postoperative intercostal neuralgia, and four patients with electrolyte disturbance, six patients with anemia and hypoproteinemia,all of which were relieved after symptomatic treatment. there was no patients with pneumothorax and no cases of cerebrospinal fluid leak. Wounds were healed without chronic infection or sinus formation. No complications related to instrumentation occurred. 16 patients had only a small amount of pleural effusion after surgery, which had been absorbed by the third month of reexamination.

\section{Discussion}


Spinal TB is one of the most common and severe forms of bone and joint TB. It mostly occurs in the thoracic spine region and always involves the anterior and middle spine. A lesion of the vertebral body caused by TB always lead to the development of kyphosis, paravertebral abscesses, or progressive neurological impairment[14]. Seriously,neurological impairment can arise from mechanical compression on the neural elements by epidural abscesses, tubercular debris,caseous and granulation tissue, or by mechanical instability produced by pathological subluxation or islocation[15]. Neurological impairment is more common in thoracic tuberculosis than in spinal TB elsewhere, due to a narrower spinal canal, physiological kyphosis in the thoracic region and greater biomechanical forces in the thoracolumbar region. Godlwana et al[16]reported thoracic spinal tuberculosis accounted for $52 \%$ of all spinal tuberculosis cases with neurological deficits. Therefore, we selected such patients for a retrospective study.The results of this study reveaed that Pott disease or incomplete paralysis is primarily caused by nerve compression by epidural abscesses, tuberculous debris, necrotic intervertebral discs, and caseous and granulomatous tissue.

Although conservative treatment for spinal TB can effectively alleviate pain, kyphotic deformity continues to progress, with $3 \%$ to $5 \%$ of patients showing severe progression and subsequent paraplegia or incomplete paralysis[17]. Although chemotherapy is a very effective way of controlling and treating the disease and is anindispensable treatment strategy [12],But it is particularly important to perform necessary surgical interventions to relieve clinical symptoms and achieve good long-term outcomes[18].

Various surgical techniques have been used to treat spinal tuberculosis, but few literatures on treating short segmental thoracic tuberculosis with paraplegia or incomplete paralysis have been reported.The goal of surgery for Pott's disease is to adequately debride the focus area, decompress the spinal cord, correct spinal deformities and rebuild spinal stability. Although several severe complications related to anterior surgical approaches to the thoracic spine have been described in the literature $[19,20]$. But it is a convenient way to completely remove tuberculotic substances from the lesion vertebra and paravertebral abscesses, to safely decompress soft oppression of the spinal cord, and to fully perform spinal canal decompression. Anterior debridement and strut grafting enable surgeons to treat thoracic spinal tuberculosis with paraplegia directly and thoroughly, which is more favorable for biomechanical reconstruction. Anterior internal fixation can still maintain the spinal stability and correction of kyphosis when tuberculosis invades short thoracic vertebra segments. The anterior approach enables surgeons to reach the lesion site directly, and a single incision can be used to perform multiple operations. Operators also have a more spacious and a direct field of vision, simplifying the operative procedures[21].Anterior debridement combined with strut grafting can provide a suitable host bed to simulate vertebral bone fusion, and instrumentation by titanium plate and mesh stabilizes biomechanical properties of the spine. This will reduce the risk of postoperative kyphosis and improve the surgical cure rate of short segmental thoracic vertebrae with paraplegia or incomplete paralysis.In our series,we carried out one-stage anterior focus debridement, interbody graft using titamium mesh cages, and anterior instrumentation and fusion in the surgical treatment of short segment thoracic tuberculosis. All patients obtained satisfactory functional restoration and recovery from short segment tuberculosis through debridement,decompression and stabilization of the spinal. 
We believe that one-stage anterior surgical indications mainly includes the damaged portion of the vertebra being located in the anterior and middle columns of the spine; the abscess or sequestrum invading the anterior spinal canal and causing compression; unapparent kyphosis; laminectomy having been performed and posterior bone graft fusion being prevented; and when the number of damaged vertebrae is less than $3[22 \llbracket 23]$.Patients who did not meet these conditions were excluded.

For interbody fusion in patients with tuberculosis spondylitis, the literature indicates that several kinds of graft materials have been used, such as autogenous rib, iliac crest,and allograft[24].The ribs are very tenuous, particularly the lower ribs. They do not provide adequate stability to the anteriorcolumn because of plastic deformation and the small surface area of contact with the adjacent normal vertebral bodies[25].Many recent studies reported that titanium mesh cages showed potential for reliable spinal reconstruction, high bone fusion,sufficient sagittal profile maintenance, and low implant-related problems[26].Titanium mesh cage was used for interbody fusion in this study. It is usually filled with healthy ribs. Making full use of autologous ribs avoids the disadvantages of interception of autologous bone from other parts and the economic burden of allograft bone. The use of titanium mesh cages has several benefits over other bone struts. The cage provides immediate stability, is rigid, and can tolerate compression forces well. The significant interface strength between the cage and endplates prevents it from extrusion or displacement[27].Most of all, the titanium mesh cage is the ideal shape,lt has relatively large weight-bearing surfaces. It is mechanicallystrong enough to prevent discrete loss of height from a fused motion segment because of steoporosis of the vertebrae[28]. All patients in our study in the titanium mesh cage group achieved bone fusion. The average fusion time was 5.3 months(range, 4-8 months), judging by $\mathrm{x}$-ray and/or CT scan.

Although it's been reported in the literature,anterior spinal surgery may suffer the disadvantages of greater surgical trauma and more complications, including vascular andvisceral injury, chylous leakage and others. Subsidence of titanium mesh cages was reported in anterior column reconstruction after anterior spine surgery[29].However, these defects did not appear during surgery, and these complications also did not appear during follow-up in our study, which may be related to our years of experience in thoracic surgery and our proficiency in open-heart surgery, or to the limited extent of damage and minor injury of short-segment vertebral tuberculosis.

Results obtained from this study showed that no serious complications occurred during the postoperative and follow-up period. No complications of atelectasis and pulmonary tuberculosis were found because perioperative nursing of thoracic surgery was strengthened. Additionally, complete focal cleaning expanded the scope of operation and allowed removal of all necrotic tissue.The compression of granulation tissue on the dural sac was relieved.We believe this also helps recovery. However, tuberculosis cure still relies on formal chemotherapy[30].

Sixteen patients of short-segment thoracic tuberculosis with paraplegia in our study underwent the anterior approach debridement, strut grafting, and instrumentation and all of them had a sturdy implant and favorable curative state. A titanium cage supplemented by autologous bone or allograft bone graft 
achieved satisfying outcomes in our study. At the last follow-up, All patients recovered well without breakage or transposition of the implant or kyphosis recurrence. and all patients had achieved bone fusion, relief from pain, and neurological recovery or significant improvement.

Although satisfactory outcomes were obtained in this research, several limitations exist. First, this was a retrospective research, with a shorter follow-up period, which may affect the reliability of the evaluation results. Second, a few eligible cases were included and clinical studies with large samples were lacking,which may cause a certain degree of bias.

\section{Conclusions}

In summary, we believe that the simple anterior approach for debridement, strut grafting, and instrumentation should be considered for tuberculosis of short segmental thoracic tuberculosis with paraplegia or incomplete paralysis. The operative method should be determined based on the specific circumstances of each patient and the operator's proficiency level. Systemic anti-tuberculosis chemotherapy is essential to cure spinal tuberculosis. Lastly, comprehensive measures must be taken to improve the cure rate of short segmental thoracic tuberculosis with paraplegia or incomplete paralysis.

\section{Declarations}

\section{Acknowledgments}

We acknowledge Huijun Zhang,Zenghuui Lu,Chao Ding and Lin Wei for their assistance with database collection.

\section{Funding}

No funds were received in support of this work.

\section{Availability of data and materials}

The datasets used and/or analyzed during the current study are available from the corresponding author on reasonable request.

\section{Abbreviations}

CRP:C-reactive protein;ESR:Erthrocyte sedimentation rate;FFU:Final followup;POST:Postoperative,PRE:Preoperative

\section{Author's contributions}

Huijun Zhang collected, analyzed, and interpreted the data and wrote the draft. Huijun Zhang and Zenghui Lu and Lin Wei performed the surgery. Lin Wei and Chao Ding assisted in the follow-up process 
and collection of data. Jun song Yang critically revised the manuscript. All the authors have read and approved the final manuscript.

\section{Ethics approval and consent to participate}

Written informed consent was obtained from all patients, and the study protocol was approved by the Institutional Ethics Review Board of XI'AN Chest Hospital.

\section{Consent for publication}

Written consent to publish patient identifiable information and data was obtained from the patients.

\section{Competing interests}

The authors declare that they have no competing interests.

\section{References}

1. Wibaux C, Moafo-Tiatsop M, Andrei I, Biver E, Cotten A, Cortet B.Changes in the incidence and management of spinal tuberculosis in a French university hospital rheumatology department from 1966 to 2010. Joint Bone Spine.2013;80(5):516-519. doi: 10.1016/j.jbspin.2012.10.001. [PubMed] [CrossRef] [Google Scholar]

2. Wang Z, Wu Q, Geng G.Anterior debridement and bone grafting with posterior single-segment internal fixation for the treatment of mono-segmental spinal tuberculosis. Injury.2013;44:253-257. [PubMed] [Google Scholar] doi: 10.1016/j.injury.2012.11.003.

3. KumarA, SahuBP.Ghost vertebra: do we need to reconsider treatment strategy for sub囚axial cervical spine tuberculosis $₫$ J Neurosci Rural Pract.2013;4(2): 237-239. DOI: 10.4103/0976-3147.112783. [PubMed] [CrossRef] [Google Scholar]

4. Kumar K. Tuberculosis of spine: natural history of disease and its judicious management. J Western Pac Orthop Assoc.1988;25:1-18.

5. Zhaohui Cheng, Jian Wang, Qixin Zheng, Yongchao Wu, Xiaodong Guo. Anterolateral Radical Debridement and Interbody Bone Grafting Combined With Transpedicle Fixation in the Treatment of Thoracolumbar Spinal Tuberculosis. Medicine (Baltimore).2015;94 (14):e721. DOI: 10.1097/MD.0000000000000721 [PubMed] [CrossRef] [Google Scholar]

6.Rajasekaran S.Natural history of Pott's kyphosis. Eur Spine J.2013;22(4):p.634-640. doi: 10.1007/s00586-012-2336-6. [PMC free article] [PubMed] [CrossRef] [Google Scholar]

7. Zeng H, Shen X, Luo C, Xu Z, Zhang Y, Liu Z.Comparison of three surgical approaches for cervicothoracic spinal tuberculosis: a retrospective case-control study. J Orthop Surg Res.2015;10:100. doi: 10.1186/s13018-015-0238-0. [PubMed] [CrossRef] [Google Scholar] 
8. LukKD.Commentary: instrumentation in the treatment of spinal tuberculosis, anterior or posterior. Spine.2011;11(8):734-736. DOI: 10.1016/j.spinee.2011.07.024. [PubMed] [CrossRef] [Google Scholar]

9. Li, ZW, Li, ZQ, Tang, BMet al.Efficacy of one-stage posterior debridement and bone grafting with internal fixation in the treatment of monosegmental thoracolumbar tuberculosis. World Neurosurg.2019;121:e843-e851. Google Scholar | Crossref | Medline doi: 10.1016/j.wneu.2018.09.234. Epub 2018 Oct

10. Wang LJ, Zhang HQ, Tang MX, Gao QL, Zhou ZH, Yin XH.Comparison of three surgical approaches for thoracic spinal tuberculosis in adult: minimum 5-year follow up. Spine.2017;42(11):808. doi: 10.1097/BRS.0000000000001955. [PubMed] [CrossRef] [Google Scholar]

11. Mihir B, Vinod L, Umesh M, Chaudhary K.Anterior instrumentation of the cervicothoracic vertebrae: approach based on clinical and radiologic criteria. Spine.2006;31(9):244-249. doi: 10.1097/01.brs.0000214883.11874.80. [PubMed] [CrossRef] [Google Scholar]

12. Zhang HQ , Lin MZ, Shen KY, Ge L, Li JS, Tang MX, Wu JH, Liu JY.Surgical management for multilevel noncontiguous thoracic spinal tuberculosis by single-stage posterior transforaminal thoracic debridement, limited decompression, interbody fusion, and posterior instrumentation (modified TTIF).Arch Orthop Trauma Surg.2012; 132(6):751-757. PMID: 22350053 DOI: 10.1007/s00402-012-1473-z[Indexed for MEDLINE]

13. Bridwell KH,Lenke LG,McEnery KW,Baldus C,Blanke K. Anetrior fresh frozen structural allografts in the thoracic and lumbar spine.Do they work if combined with posterior fusion and instrumentation in adult patients with kyphosis or anterior column defects? Spine(Phila Pa 1976).1995;20:1410-1418.

14. Haiping Liu, Jing Luo, Xiaodong Wang, Xiaohua Dong, Dingjun Hao. Efficacy of Surgery via the Single Anterior and Single Posterior Approaches for Treatment of Thoracic Spinal Tuberculosis. J Int Med Res.2020;48 (1): 1-11. PMID: 31937167 DOI: 10.1177/0300060519896743 [PubMed] [CrossRef] [Google Scholar]

15. Jain AK. Tuberculosis of the spine: a fresh look at an old disease. J Bone Joint Surg Br.2010;92:90513.

16. Godlwana L, Gounden P, Ngubo P, et al. Incidence and profile of spinal tuberculosis in patients at the only public hospital admitting such patients in KwaZulu-Natal. Spinal Cord.2008;46:372-4.

17. Rajasekaran, S, Soundararajan, DCR, Shetty, APet al.Spinal tuberculosis: current concepts. Global Spine J.2018;8: 96S-108S. doi: 10.1177/2192568218769053. Epub 2018 Dec 13.Google Scholar | SAGE Journals | ISI

18. Dunn, RN, Ben Husien, M.Spinal tuberculosis: review of current management. Bone Joint J.2018;100B:425-431.doi: 10.1302/0301-620X.100B4.BJJ-2017-1040.R1. Google Scholar | Crossref | Medline 19. Tuli SM.Tuberculosis of the spine: a historical review. Clin Orthop Relat Res.2007; 460:29-38. 
20. Pointillart V, Aurouer N, Gangnet $\mathrm{N}$ et al.Anterior approach to the cervicothoracic junction without sternotomy: a report of 37 cases. Spine.2007;32:2875-2879.

21. Benli IT, Kaya A, Acaroğlu E.Anterior instrumentation in tuberculous spondylitis: is it effective and safe? Clin Orthop Relat Res.2007;460(460):108-116. [PubMed] [Google Scholar] PMID: 17452918 DOI: 10.1097/BLO.0b013e318065b70d

22. Przybylski GJ, Sharan AD.Single-stage autogenous bone grafting and internal fixation in the surgical management of pyogenic discitis and vertebral osteomyelitis. J Neurosurg. 2001;94:1-7.

23. Zhang H, Zeng K, Yin X, Huang J, Tang M, Guo C.Debridement, internal fixation, and reconstruction using titanium mesh for the surgical treatment of thoracic and lumbar spinal tuberculosis via a posterior only approach: A 4-year follow-up of 28 patients. J Orthop Surg Res. 2015;10:1-9.

24. Govender S, Kumar KP. Cortical allografts in spinal tuberculosis. Int Orthop. 2003;27:244-248.

25. Govender S, Parbhoo AH. Support of the anterior column with allografts in tuberculosis of the spine. J Bone Joint Surg Br.1999;81:106-109.

26. Sundararaj GD, Amritanand R, Venkatesh K, Arockiaraj J. The use of titanium mesh cages in the reconstruction of anterior column defects in active spinal infections: Can we rest the crest?Asian Spine J. 2011;5:155-161.

27. Zhang HQ, Li M, Wang YX, Tang MX, Guo CF, Liu SH, Deng A, Gao Q. Minimum 5-Year Follow-Up Outcomes for Comparison Between Titanium Mesh Cage and Allogeneic Bone Graft to Reconstruct Anterior Column Through Posterior Approach for the Surgical treatment of Thoracolumbar Spinal Tuberculosis with Kyphosis.World Neurosurg. 2019;127:e407-e415. doi: 10.1016/j.wneu.2019.03.139. Epub 2019 Mar 23.

28. Pee YH, Park JD, Choi YG, Lee SH.Anterior debridement and fusion followed by posterior pedicle screw fixation in pyogenic spondylodiscitis: autologous iliac bone strut versus cage. J Neurosurg Spine.2008;8(5):405-412. doi:10.3171/SPI/2008/8/5/405

29. Dvorak MF, Kwon BK, Fisher CG, et al.Effectiveness of titanium mesh cylindrical cages in anterior column reconstruction after thoracic and lumbar vertebral body resection. Spine.2003;28:902-8.

30. LoBue PA, Enarson DA, Thoen TC.Tuberculosis in humans and its epidemiology, diagnosis and treatment in the United States. Int J Tuberc Lung Dis.2010 14:1226-1232.

\section{Figures}



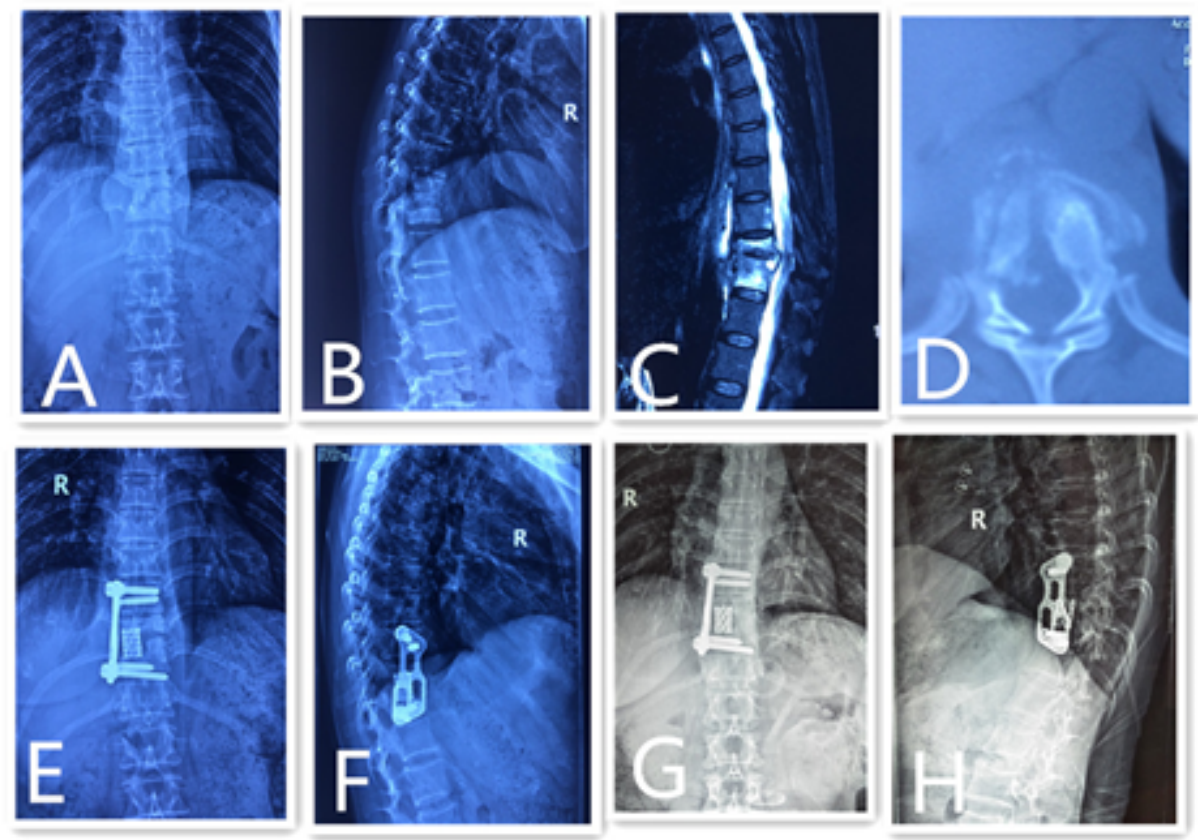

\section{Figure 1}

Forty-seven-year-old female with short segment thoracic vertebra tuberculosis (T10-11 level). A,B Preoperative frontal and lateral $x$-rays showed bone destruction in the 10/11 vertebral body and narrowing of the vertebral space $\mathrm{C}$ Preoperative magnetic resonance imaging scan $\mathrm{D}$ Preoperative computed tomography scan demonstrate vertebral body destruction. E,F Radiographs postoperatively showing well-positioned internal fixation. G,H Radiographs showing satisfactory focal clearance and strut graft stability at the final follow-up
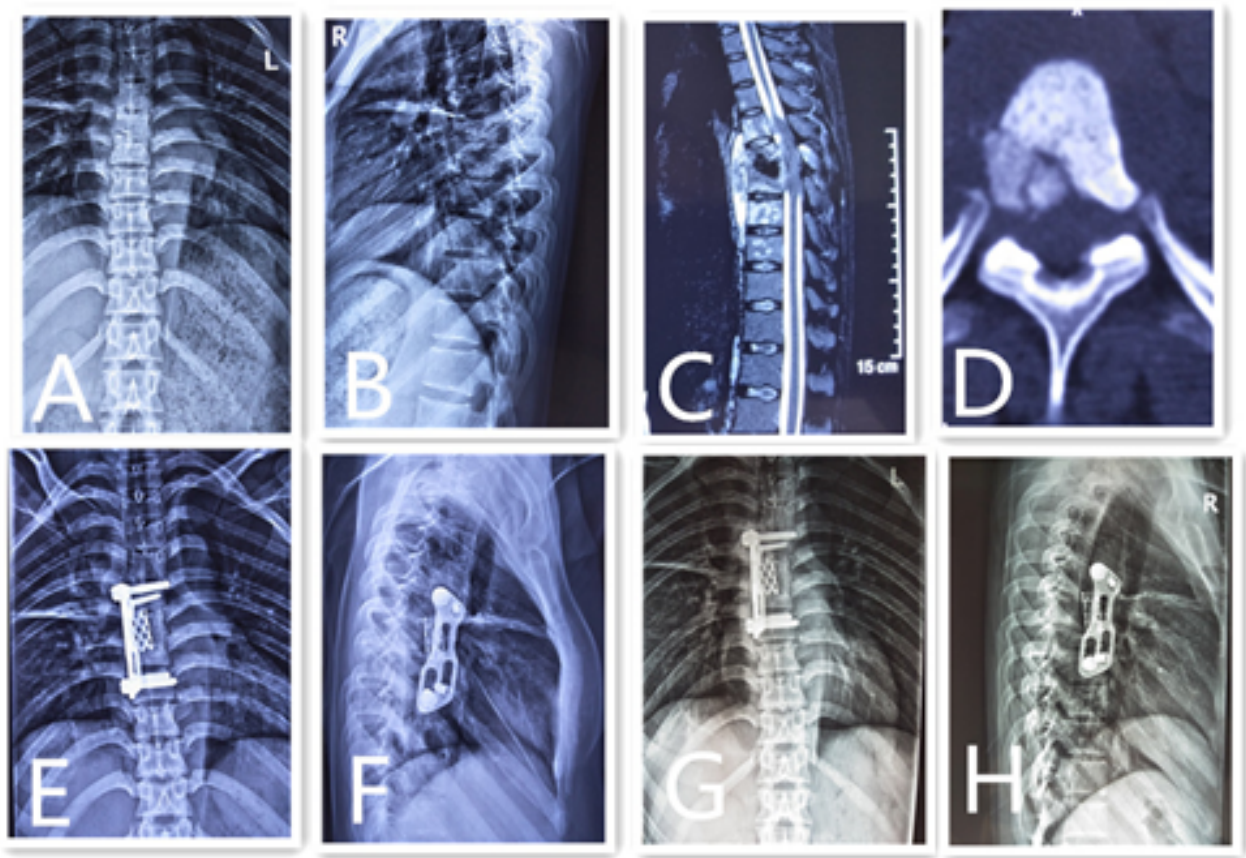

Figure 2 
Twenty-six-year-old girl with short segment thoracic vertebra tuberculosis (T6-T7 level). A,B Preoperative frontal and lateral $x$-rays showed bone destruction in the $6 / 7$ vertebral body and narrowing of the vertebral space $C$ Preoperative magnetic resonance imaging scan shows vertebral body destruction and abscess. D Preoperative computed tomography scans demonstrate vertebral body destruction. E,F Radiographs postoperatively showing well-positioned internal fixation. G,H Radiographs showing satisfactory focal clearance and strut graft stability at the final follow-up
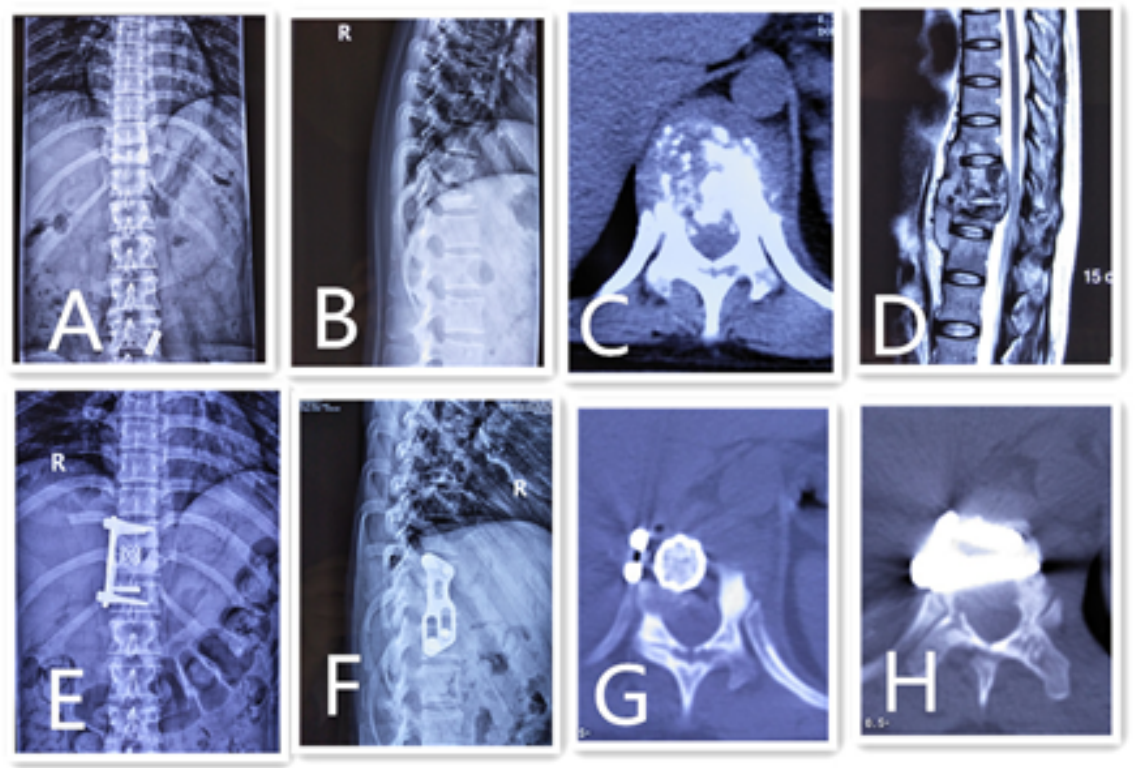

\section{Figure 3}

Thity-two-year-old girl with short segment thoracic vertebra tuberculosis (T10-T11 level). A,B Preoperative frontal and lateral x-rays showed bone destruction in the 10/11 vertebral body and narrowing of the vertebral space $C$ Preoperative computed tomography scans demonstrate vertebral body destruction. $D$ Preoperative magnetic resonance imaging scan shows vertebral body destruction and abscess. The lesion compressed the dural sac. E,F Radiographs postoperatively showing well-positioned internal fixation and Kyphotic deformity was corrected. G,H Postoperative axial CT showed satisfactory internal fixation device at the final follow-up 